\title{
Obstetrics and Gynaecology
}

Acta Genet Med Gemellol 43: 113-114 (1994)

(C) 1994 by The Mendel Institute

International Congress

40th Anniversary of The Mendel Institute

\section{Gravidanze Gemellari in 18 Anni di Diagnostica Citogenetica Prenatale}

\author{
M.M. Mantero, G. Piombo, F. Zara, L. Doria Lamba Carbone \\ Centro Genetica Umana, E.O. Ospedali Galliera, Genova, Italia
}

\begin{abstract}
Riportiamo 55 casi di gravidanze gemellari su un totale di 7,350 (0.75\%) diagnosi citogenetiche prenatali eseguite presso il Centro di Genetica Umana dell'E.O. Ospedali Galliera di Genova, nel periodo 1975-1993. Le gravidanze gemellari erano biamniotiche nel $93.7 \%$ dei casi e monoamniotiche nel restante $6.3 \%$. Le indicazioni per cui è stata richiesta la diagnostica citogenetica prenatale sono riportate in Tab. I
\end{abstract}

Tabella I

\begin{tabular}{lrr}
\hline Indicazione & N. & \multicolumn{1}{c}{$\%$} \\
\hline Età materna $\geq 35$ & 47 & 85.5 \\
Precedente figlio affetto da anomalia cromosomica & 1 & 1.8 \\
Genitore portatore di riarrangiamento strutt. bil. & 1 & 1.8 \\
Miscellanea & 6 & 10.9 \\
\hline Totale & $\mathbf{5 5}$ & $\mathbf{1 0 0}$ \\
\hline
\end{tabular}

La quasi totalità delle diagnosi (n. 54) è stata effettuata mediante prelievo di liquido amniotico tra la $15 \mathrm{ma}$ e la $21 \mathrm{ma}$ settimana di gestazione; in un solo caso è stato richiesto il prelievo dei villi coriali alla $11 \mathrm{ma}$ settimana. In Tab. II riportiamo i sessi fetali riscontrati.

\section{Tabella II}

\begin{tabular}{cccc}
\hline FF & MM & MF & TOTALE \\
\hline 19 & 16 & 20 & 55 \\
\hline
\end{tabular}




\section{M.M. Mantero et al.}

In n. 3 gravidanze $(5.5 \%)$ è stato evidenziato un cariotipo fetale patologico.

1) $46, X Y$

47, XXY

2) $47, X Y,+18$

$47, X Y,+18$

3) $46, X Y, t(9 ; 15)(p 22 ; q 26)$ mat

$46, \mathrm{XY}, \mathrm{t}(9 ; 12)(\mathrm{p} 22 ; \mathrm{q} 26)$ mat

- nei casi 1) e 2) le gravidanze sono state interrotte per scelta dei genitori mediante aborto elettivo.

- nel caso 3) la gravidanza è esitata in parto prematuro alla 29 ma settimana di gestazione; uno dei due gemelli, risultato già all'esame ecografico in $15 \mathrm{ma}$ settimana affetto da anencefalia, è deceduto poco dopo la nascita, l'altro attualmente, all'età di 2 anni, presenta normale sviluppo staturo-ponderale e psicomotorio.

In due casi, per la mancata evidenziazione ecografica della gemellarità, è stato dato il referto citogenetico su di un solo gemello.

\section{(Presentato come poster)}

Per Corrispondenza: Dr. G. Piombo, Centro Genetica Umana, E.O. Ospedali Galliera, Via Volta 8, 16128 Genova, Italia. 